\title{
Highlighting Resources to Study Cardiovascular Disease and Diabetes in US Immigrants
}

\author{
Reena Oza-Frank ${ }^{1}$ and Solveig A. Cunningham ${ }^{2}$ \\ ${ }^{1}$ The Research Institute at Nationwide Children's Hospital and the Department of Pediatrics, The Ohio State University, \\ Columbus, $\mathrm{OH} 43205$, USA \\ ${ }^{2}$ Hubert Department of Global Health, Rollins School of Public Health, Emory University, Atlanta, GA 30322, USA
}

Correspondence should be addressed to Reena Oza-Frank, oza-frank.1@osu.edu

Received 14 November 2011; Accepted 22 December 2011

Academic Editors: M. Askarian, S. Gallus, and E. Lazcano-Ponce

Copyright ( $) 2012$ R. Oza-Frank and S. A. Cunningham. This is an open access article distributed under the Creative Commons Attribution License, which permits unrestricted use, distribution, and reproduction in any medium, provided the original work is properly cited.

\begin{abstract}
International migration is at an all-time high, with the largest numbers migrating to the US, making this an ideal setting to study migrant health. While previous studies have shown that foreign-born individuals in the US tend to be in better health than the native born with regard to several health outcomes, less is known about such advantages in terms of cardiovascular disease and diabetes, which are some of the leading causes of morbidity and mortality worldwide. In this paper, we highlight the main existing resources available for the study of cardiometabolic health among US immigrants that researchers have yet to fully utilize. Despite the limited literature on migration and cardiovascular disease and diabetes, there are several data sources that can be used to better understand the importance of location exposures and of predispositions to these diseases.
\end{abstract}

\section{Introduction}

Cardiovascular disease (CVD) is the leading cause of death worldwide and is projected to remain so through at least 2015 [1]. Type 2 diabetes (T2D) is a leading cause of morbidity as well as a risk factor for CVD. Currently, 246 million people are estimated to have T2D worldwide, and almost 4 million deaths are attributable to diabetes each year [2].

At the same time, international migration is at an all time high [3]. Over 191 million individuals migrated worldwide in 2005 [3]. Yet, little is known about the patterns of CVD and T2D among migrants. The US receives more immigrants than any other nation [4]: in 2006, 1.2 million migrants entered the US [3]. Over $12 \%$ of the US population is currently foreign born [5], the largest proportion since the early 1900s. As the migrant population continues to increase in size and diversify, researchers have an opportunity to study CVD and T2D among migrants taking advantage of potentially large sample sizes, including in existing datasets. Research on health patterns among immigrants provides critical information for improving health systems. It can also help to explicate the genetic and environmental determinants of disease worldwide by examining the importance of place exposures and variations in predispositions.

The few studies that have examined CVD and T2D among immigrants in the US have found that although immigrants tend to be in better health by most measures, their advantages in terms of CVD and T2D are less consistent $[6,7]$. A majority of studies find T2D prevalence to be lower among the foreign-born compared with the native-born population. However, these risks may differ by place of origin between by race and ethnic groups [6]. While foreign-born individuals may share characteristics with others of the same race or ethnicity, they also share important characteristics with each other, including the migration process itself [8].

Reviews of the literature on cardiometabolic health among foreign-born individuals in the US is multidisciplinary but limited, often reporting inconsistent associations across subpopulations [6]. Research gaps remain to be filled, making it difficult to assess the factors associated with cardiometabolic health among immigrants. This review highlights the main available data sources that can be used to extend our understanding of cardiometabolic health among immigrants. It presents a list of datasets from 
TABLE 1: Existing datasets incorporating variables of migration and cardiometabolic health.

\begin{tabular}{|c|c|c|c|c|c|c|c|c|}
\hline \multicolumn{5}{|c|}{ Study information } & \multicolumn{4}{|c|}{ Migration variables } \\
\hline Study title & Study type & $\begin{array}{l}\text { Study start } \\
\text { date }\end{array}$ & $\begin{array}{l}\text { Age } \\
\text { range }\end{array}$ & Races/ethnicities & $\begin{array}{l}\text { Country } \\
\text { of birth }\end{array}$ & $\begin{array}{l}\text { Year } \\
\text { came } \\
\text { to US }\end{array}$ & $\begin{array}{l}\text { Duration } \\
\text { of } \\
\text { residence } \\
\text { in US }\end{array}$ & $\begin{array}{l}\text { Parent's } \\
\text { place of } \\
\text { birth }\end{array}$ \\
\hline $\begin{array}{l}\text { California Health Interview Survey } \\
\text { (CHIS) }\end{array}$ & $\begin{array}{l}\text { Serial cross- } \\
\text { sectional }\end{array}$ & 2001 & All & All & & & & \\
\hline Charleston Heart Study & Longitudinal & 1960 & $35+$ & White and Black & & & & \\
\hline $\begin{array}{l}\text { Diabetes Study of Northern } \\
\text { California (DISTANCE) Survey }\end{array}$ & Longitudinal & 2005 & $50-75$ & All & & & & \\
\hline $\begin{array}{l}\text { Early Childhood Longitudinal } \\
\text { Study-Kindergarten cohort } \\
\left(\text { ECLS-K) }{ }^{\mathrm{a}}\right.\end{array}$ & Longitudinal & 1998 & $5-14$ & All & & & & \\
\hline $\begin{array}{l}\text { Health and Retirement Study } \\
(\text { HRS })^{\mathrm{a}}\end{array}$ & Longitudinal & 1992 & $50+$ & All & & & & \\
\hline $\begin{array}{l}\text { Hispanic Community Health } \\
\text { Study-Study of Latinos } \\
\text { (HCHS-SOL) }\end{array}$ & Longitudinal & 2006 & $18-74$ & $\begin{array}{l}\text { Cuban, Puerto } \\
\text { Rican, } \\
\text { Central/South } \\
\text { American, } \\
\text { Mexican American }\end{array}$ & & & & \\
\hline $\begin{array}{l}\text { Hispanic Health and Nutrition } \\
\text { Examination Survey (HHANES) }\end{array}$ & Cross sectional & 1982 & $\begin{array}{l}6 \text { mos. } \\
-74 \text { yrs }\end{array}$ & Hispanics & & & & \\
\hline Honolulu Heart Study & Longitudinal & 1965 & $45-68$ & Japanese & & & & \\
\hline $\begin{array}{l}\text { Japanese American Community } \\
\text { Diabetes Study }\end{array}$ & Longitudinal & 1983 & $34-75$ & Japanese & & & & \\
\hline The Konkani Heart Study & Cross sectional & 2003 & $18+$ & $\begin{array}{l}\text { Konkani (South } \\
\text { Indian) }\end{array}$ & & & & \\
\hline Masala Study & Cross sectional & 2006 & $45-79$ & Asian Indian & & & & \\
\hline Mexican Family Life Survey & Longitudinal & 2002 & $15+$ & Mexicans & & & & \\
\hline $\begin{array}{l}\text { Multiethnic Study of } \\
\text { Atherosclerosis (MESA) }\end{array}$ & Longitudinal & 2000 & $45-84$ & All & & & & \\
\hline $\begin{array}{l}\text { National Longitudinal Study of } \\
\text { Adolescent Health (Add Health) })^{\mathrm{a}, \mathrm{b}, \mathrm{c}}\end{array}$ & Longitudinal & 1994 & $12-18$ & All & & & & \\
\hline New Immigrant Survey (NIS) $)^{\mathrm{a}, \mathrm{b}, \mathrm{d}}$ & Longitudinal & $\begin{array}{l}2003 \text { (pilot } \\
1996)\end{array}$ & All & All & & & & \\
\hline $\begin{array}{l}\text { National Health and Nutrition } \\
\text { Examination Survey (NHANES) }{ }^{\mathrm{a}, \mathrm{e}}\end{array}$ & $\begin{array}{l}\text { Serial cross } \\
\text { sectional }\end{array}$ & 1971 & All & All & & & & \\
\hline $\begin{array}{l}\text { National Health Interview Survey } \\
(\text { NHIS })^{\mathrm{a}, \mathrm{c}}\end{array}$ & $\begin{array}{l}\text { Serial cross } \\
\text { sectional }\end{array}$ & 1957 & All & All & & & & \\
\hline $\begin{array}{l}\text { National Longitudinal Survey of } \\
\text { Youth (NLSY) }\end{array}$ & Longitudinal & 1979 & $14-22$ & All & & & & \\
\hline Rancho Bernardo Study & Longitudinal & 1972 & $20+$ & Caucasian & & & & \\
\hline San Antonio Family Heart Study & Longitudinal & 1979 & $25-64$ & $\begin{array}{l}\text { Mexican American } \\
\text { and non-Hispanic } \\
\text { White }\end{array}$ & & & & \\
\hline San Luis Valley Diabetes Study & Longitudinal & 1984 & $20-74$ & $\begin{array}{l}\text { Hispanics and } \\
\text { non-Hispanic } \\
\text { Whites }\end{array}$ & & & & \\
\hline
\end{tabular}


Table 1: Continued.

\begin{tabular}{|c|c|c|c|c|c|c|c|c|}
\hline \multicolumn{5}{|c|}{ Study information } & \multicolumn{4}{|c|}{ Migration variables } \\
\hline Study title & Study type & $\begin{array}{c}\text { Study start } \\
\text { date }\end{array}$ & Age range & Races/ethnicities & $\begin{array}{c}\text { Country } \\
\text { of } \\
\text { birth }\end{array}$ & $\begin{array}{l}\text { Year } \\
\text { came } \\
\text { to US }\end{array}$ & $\begin{array}{l}\text { Duration } \\
\text { of } \\
\text { residence } \\
\text { in US }\end{array}$ & $\begin{array}{l}\text { Parent's } \\
\text { place of } \\
\text { birth }\end{array}$ \\
\hline U.S. Mexico Borders Study & Cross Sectional & 2001 & $18+$ & Mexicans & & & & \\
\hline $\begin{array}{l}\text { University of California San } \\
\text { Diego Filipino Health Study }\end{array}$ & Longitudinal & 1995 & $40-86$ & Filipino & & & & \\
\hline \multicolumn{5}{|c|}{ Study information } & \multicolumn{4}{|c|}{ Anthropometric variables } \\
\hline Study title & Study type & $\begin{array}{c}\text { Study start } \\
\text { date }\end{array}$ & Age range & Races/ethnicities & Height & Weight & \begin{tabular}{c|} 
Waist \\
circumference
\end{tabular} & $\begin{array}{l}\text { Body } \\
\text { fat } \%\end{array}$ \\
\hline $\begin{array}{l}\text { California Health Interview } \\
\text { Survey (CHIS) }\end{array}$ & $\begin{array}{l}\text { Serial cross } \\
\text { sectional }\end{array}$ & 2001 & All & All & SR & SR & & \\
\hline Charleston Heart Study & Longitudinal & 1960 & $35+$ & White and Black & & & & \\
\hline $\begin{array}{l}\text { Diabetes Study of Northern } \\
\text { California (DISTANCE) Survey }\end{array}$ & Longitudinal & 2005 & $50-75$ & All & & & & \\
\hline $\begin{array}{l}\text { Early Childhood Longitudinal } \\
\text { Study-Kindergarten cohort } \\
(\text { ECLS-K) })^{\mathrm{a}}\end{array}$ & Longitudinal & 1998 & $5-14$ & All & & & & \\
\hline $\begin{array}{l}\text { Health and Retirement Study } \\
(\text { HRS })^{\mathrm{a}}\end{array}$ & Longitudinal & 1992 & $50+$ & All & & & & \\
\hline $\begin{array}{l}\text { Hispanic Community Health } \\
\text { Study-Study of Latinos } \\
\text { (HCHS-SOL) }\end{array}$ & Longitudinal & 2006 & $18-74$ & $\begin{array}{l}\text { Cuban, Puerto } \\
\text { Rican, } \\
\text { Central/South } \\
\text { American, } \\
\text { Mexican American } \\
\end{array}$ & & & & \\
\hline $\begin{array}{l}\text { Hispanic Health and Nutrition } \\
\text { Examination Survey (HHANES) }\end{array}$ & Cross sectional & 1982 & $\begin{array}{c}6 \text { mos-74 } \\
\text { yrs }\end{array}$ & Hispanics & & & & \\
\hline Honolulu Heart Study & Longitudinal & 1965 & $45-68$ & Japanese & & & & \\
\hline $\begin{array}{l}\text { Japanese American Community } \\
\text { Diabetes Study }\end{array}$ & Longitudinal & 1983 & $34-75$ & Japanese & & & & \\
\hline The Konkani Heart Study & Cross sectional & 2003 & $18+$ & $\begin{array}{l}\text { Konkani (South } \\
\text { Indian) }\end{array}$ & & & & \\
\hline Masala Study & Cross sectional & 2006 & $45-79$ & Asian Indian & & & & \\
\hline Mexican Family Life Survey & Longitudinal & 2002 & $15+$ & Mexicans & & & & \\
\hline $\begin{array}{l}\text { Multiethnic Study of } \\
\text { Atherosclerosis (MESA) }\end{array}$ & Longitudinal & 2000 & $45-84$ & All & & & & \\
\hline $\begin{array}{l}\text { National Longitudinal Study of } \\
\text { Adolescent Health (Add } \\
\text { Health) })^{\mathrm{a}, \mathrm{b}, \mathrm{c}}\end{array}$ & Longitudinal & 1994 & $12-18$ & All & & & & \\
\hline New Immigrant Survey (NIS) $)^{\mathrm{a}, \mathrm{b}, \mathrm{d}}$ & Longitudinal & $\begin{array}{c}2003 \text { (pilot } \\
1996) \\
\end{array}$ & All & All & SR & SR & & \\
\hline $\begin{array}{l}\text { National Health and Nutrition } \\
\text { Examination Survey } \\
\left(_{\text {NHANES })^{\mathrm{a}, \mathrm{e}}}\right.\end{array}$ & $\begin{array}{l}\text { Serial cross } \\
\text { sectional }\end{array}$ & 1971 & All & All & & & & \\
\hline $\begin{array}{l}\text { National Health Interview } \\
\text { Survey (NHIS) }{ }^{\mathrm{a}, \mathrm{c}}\end{array}$ & $\begin{array}{l}\text { Serial cross } \\
\text { sectional }\end{array}$ & 1957 & All & All & SR & SR & & \\
\hline $\begin{array}{l}\text { National Longitudinal Survey of } \\
\text { Youth (NLSY) }\end{array}$ & Longitudinal & 1979 & $14-22$ & All & SR & SR & & \\
\hline Rancho Bernardo Study & Longitudinal & 1972 & $20+$ & Caucasian & & & & \\
\hline
\end{tabular}


Table 1: Continued.

\begin{tabular}{|c|c|c|c|c|c|c|c|c|c|}
\hline \multicolumn{5}{|c|}{ Study information } & \multicolumn{5}{|c|}{ Anthropometric variables } \\
\hline Study title & Study type & $\begin{array}{c}\text { Study start } \\
\text { date }\end{array}$ & Age range & Races/ethnicities & Height $\mathrm{V}$ & Weight & \multicolumn{2}{|c|}{\begin{tabular}{|c|} 
Waist \\
circumference
\end{tabular}} & $\begin{array}{l}\text { Body } \\
\text { fat } \%\end{array}$ \\
\hline San Antonio Family Heart Study & Longitudinal & 1979 & $25-64$ & $\begin{array}{l}\text { Mexican American } \\
\text { and non-Hispanic } \\
\text { White }\end{array}$ & & & & & \\
\hline San Luis Valley Diabetes Study & Longitudinal & 1984 & $20-74$ & $\begin{array}{l}\text { Hispanics and } \\
\text { non-Hispanic } \\
\text { Whites }\end{array}$ & & & & & \\
\hline U.S. Mexico Borders Study & Cross sectional & 2001 & $18+$ & Mexicans & & & & & \\
\hline $\begin{array}{l}\text { University of California San } \\
\text { Diego Filipino Health Study }\end{array}$ & Longitudinal & 1995 & $40-86$ & Filipino & & & & & \\
\hline \multicolumn{5}{|c|}{ Study information } & \multicolumn{5}{|c|}{ Cardiovascular variables $^{\mathrm{h}}$} \\
\hline Study title & Study type & $\begin{array}{c}\text { Study start } \\
\text { date }\end{array}$ & Age range & Races/ethnicities & $\begin{array}{l}\text { Blood } \\
\text { pressure }\end{array}$ & cho & $\begin{array}{l}\text { Total } \\
\text { olesterol }\end{array}$ & $\begin{array}{l}\text { LDL, } \\
\text { trigly }\end{array}$ & $\begin{array}{l}\text { HDL, } \\
\text { erides }\end{array}$ \\
\hline $\begin{array}{l}\text { California Health Interview Survey } \\
\text { (CHIS) }\end{array}$ & $\begin{array}{l}\text { Serial cross } \\
\text { sectional }\end{array}$ & 2001 & All & All & SR & & SR & & \\
\hline Charleston Heart Study & Longitudinal & 1960 & $35+$ & White and Black & & & & & \\
\hline $\begin{array}{l}\text { Diabetes Study of Northern } \\
\text { California (DISTANCE) Survey }\end{array}$ & Longitudinal & 2005 & $50-75$ & All & & & & & \\
\hline $\begin{array}{l}\text { Early Childhood Longitudinal } \\
\text { Study-Kindergarten cohort } \\
(\text { ECLS-K) })^{\mathrm{a}}\end{array}$ & Longitudinal & 1998 & $5-14$ & All & & & & & \\
\hline $\begin{array}{l}\text { Health and Retirement Study } \\
(\text { HRS })^{\mathrm{a}}\end{array}$ & Longitudinal & 1992 & $50+$ & All & & & & & \\
\hline $\begin{array}{l}\text { Hispanic Community Health } \\
\text { Study-Study of } \\
\text { Latinos (HCHS-SOL) }\end{array}$ & Longitudinal & 2006 & $18-74$ & \begin{tabular}{|l|} 
Cuban, Puerto \\
Rican, \\
Central/South \\
American, Mexican \\
American \\
\end{tabular} & & & & & \\
\hline $\begin{array}{l}\text { Hispanic Health and Nutrition } \\
\text { Examination Survey (HHANES) }\end{array}$ & Cross sectional & 1982 & $\begin{array}{c}6 \text { mos-74 } \\
\text { yrs }\end{array}$ & Hispanics & & & & & \\
\hline Honolulu Heart Study & Longitudinal & 1965 & $45-68$ & Japanese & & & & & \\
\hline $\begin{array}{l}\text { Japanese American Community } \\
\text { Diabetes Study }\end{array}$ & Longitudinal & 1983 & $34-75$ & Japanese & & & & & \\
\hline The Konkani Heart Study & Cross sectional & 2003 & $18+$ & $\begin{array}{l}\text { Konkani (South } \\
\text { Indian) }\end{array}$ & & & & & \\
\hline Masala Study & Cross sectional & 2006 & $45-79$ & Asian Indian & & & & & \\
\hline Mexican Family Life Survey & Longitudinal & 2002 & $15+$ & Mexicans & & & & & \\
\hline $\begin{array}{l}\text { Multiethnic Study of } \\
\text { Atherosclerosis (MESA) }\end{array}$ & Longitudinal & 2000 & $45-84$ & All & & & & & \\
\hline $\begin{array}{l}\text { National Longitudinal Study of } \\
\text { Adolescent Health (Add Health) }{ }^{\mathrm{a}, \mathrm{b}, \mathrm{c}}\end{array}$ & Longitudinal & 1994 & $12-18$ & All & & & & & \\
\hline New Immigrant Survey (NIS) $)^{\mathrm{a}, \mathrm{b}, \mathrm{d}}$ & Longitudinal & $\begin{array}{c}2003 \text { (pilot } \\
1996 \text { ) }\end{array}$ & All & All & SR & & SR & & \\
\hline $\begin{array}{l}\text { National Health and Nutrition } \\
\text { Examination Survey (NHANES) }{ }^{\mathrm{a}, \mathrm{e}}\end{array}$ & $\begin{array}{c}\text { Serial cross } \\
\text { sectional }\end{array}$ & 1971 & All & All & & & & & \\
\hline $\begin{array}{l}\text { National Health Interview Survey } \\
\text { (NHIS) })^{\mathrm{a}, \mathrm{c}}\end{array}$ & $\begin{array}{l}\text { Serial cross } \\
\text { sectional }\end{array}$ & 1957 & All & All & SR & & SR & & \\
\hline
\end{tabular}


Table 1: Continued.

\begin{tabular}{|c|c|c|c|c|c|c|c|c|c|}
\hline \multicolumn{5}{|c|}{ Study Information } & \multicolumn{5}{|c|}{ Cardiovascular Variables $^{\mathrm{h}}$} \\
\hline Study Title & Study Type & $\begin{array}{c}\text { Study Start } \\
\text { Date }\end{array}$ & Age Range & Races/Ethnicities & $\begin{array}{c}\text { Blood } \\
\text { Pressure }\end{array}$ & \multicolumn{2}{|c|}{$\begin{array}{c}\text { Total } \\
\text { Cholesterol }\end{array}$} & \multicolumn{2}{|c|}{$\begin{array}{l}\text { LDL, HDL, } \\
\text { Triglycerides }\end{array}$} \\
\hline $\begin{array}{l}\text { National Longitudinal Survey Of } \\
\text { Youth (NLSY)a }\end{array}$ & Longitudinal & 1979 & $14-22$ & All & & & & & \\
\hline Rancho Bernardo Study & Longitudinal & 1972 & $20+$ & Caucasian & & & & & \\
\hline San Antonio Family Heart Study & Longitudinal & 1979 & $25-64$ & $\begin{array}{l}\text { Mexican American } \\
\text { and Non-Hispanic } \\
\text { White }\end{array}$ & & & & & \\
\hline San Luis Valley Diabetes Study & Longitudinal & 1984 & $20-74$ & $\begin{array}{l}\text { Hispanics and } \\
\text { Non-Hispanic } \\
\text { Whites }\end{array}$ & & & & & \\
\hline U.S. Mexico Borders Study & Cross Sectional & 2001 & $18+$ & Mexicans & & & & & \\
\hline $\begin{array}{l}\text { University of California San } \\
\text { Diego Filipino Health Study }\end{array}$ & Longitudinal & 1995 & $40-86$ & Filipino & & & & & \\
\hline \multicolumn{5}{|c|}{ Study Information } & \multicolumn{5}{|c|}{ Metabolic Variables ${ }^{i}$} \\
\hline Study Title & Study Type & $\begin{array}{c}\text { Study Start } \\
\text { Date }\end{array}$ & $\begin{array}{l}\text { Age } \\
\text { Range }\end{array}$ & Races/Ethnicities & $\begin{array}{l}\text { Fasting } \\
\text { Plasma } \\
\text { Glucose }\end{array}$ & OGTT & & $\begin{array}{l}\text { ting } \\
\text { ulin }\end{array}$ & HbAlc \\
\hline $\begin{array}{l}\text { California Health Interview } \\
\text { Survey (CHIS) }\end{array}$ & $\begin{array}{l}\text { Serial cross } \\
\text { sectional }\end{array}$ & 2001 & All & All & SR & & & & \\
\hline Charleston Heart Study & Longitudinal & 1960 & $35+$ & White and Black & & & & & \\
\hline $\begin{array}{l}\text { Diabetes Study of Northern } \\
\text { California (DISTANCE) Survey }\end{array}$ & Longitudinal & 2005 & $50-75$ & All & & & & & \\
\hline $\begin{array}{l}\text { Early Childhood Longitudinal } \\
\text { Study-Kindergarten cohort } \\
(\text { ECLS-K) }\end{array}$ & Longitudinal & 1998 & $5-14$ & All & & & & & \\
\hline $\begin{array}{l}\text { Health and Retirement Study } \\
(\text { HRS })^{\mathrm{a}}\end{array}$ & Longitudinal & 1992 & $50+$ & All & & & & & \\
\hline $\begin{array}{l}\text { Hispanic Community Health } \\
\text { Study-Study of } \\
\text { Latinos(HCHS-SOL) }\end{array}$ & Longitudinal & 2006 & $18-74$ & $\begin{array}{l}\text { Cuban, Puerto } \\
\text { Rican, } \\
\text { Central/South } \\
\text { American, Mexican } \\
\text { American } \\
\end{array}$ & & & & & \\
\hline $\begin{array}{l}\text { Hispanic Health and Nutrition } \\
\text { Examination Survey (HHANES) }\end{array}$ & Cross sectional & 1982 & $\begin{array}{l}6 \text { mos. } \\
-74 \text { yrs }\end{array}$ & Hispanics & & & & & \\
\hline Honolulu Heart Study & Longitudinal & 1965 & $45-68$ & Japanese & & & & & \\
\hline $\begin{array}{l}\text { Japanese American Community } \\
\text { Diabetes Study }\end{array}$ & Longitudinal & 1983 & $34-75$ & Japanese & & & & & \\
\hline The Konkani Heart Study & Cross Sectional & 2003 & $18+$ & $\begin{array}{l}\text { Konkani (South } \\
\text { Indian) }\end{array}$ & & & & & \\
\hline Masala Study & Cross Sectional & 2006 & $45-79$ & Asian Indian & & & & & \\
\hline Mexican Family Life Survey & Longitudinal & 2002 & $15+$ & Mexicans & & & & & \\
\hline $\begin{array}{l}\text { Multi-Ethnic Study of } \\
\text { Atherosclerosis (MESA) }\end{array}$ & Longitudinal & 2000 & $45-84$ & All & & & & & \\
\hline $\begin{array}{l}\text { National Longitudinal Study of } \\
\text { Adolescent Health (Add } \\
\text { Health) })^{\mathrm{a}, \mathrm{b}, \mathrm{c}}\end{array}$ & Longitudinal & 1994 & $12-18$ & All & & & & & \\
\hline New Immigrant Survey (NIS) $)^{\mathrm{a}, \mathrm{b}, \mathrm{d}}$ & Longitudinal & $\begin{array}{c}2003 \text { (pilot } \\
1996)\end{array}$ & All & All & SR & & & & \\
\hline
\end{tabular}


TABLE 1: Continued.

\begin{tabular}{|c|c|c|c|c|c|c|c|c|}
\hline \multicolumn{5}{|c|}{ Study information } & \multicolumn{4}{|c|}{ Metabolic variables ${ }^{\mathrm{i}}$} \\
\hline Study title & Study type & $\begin{array}{l}\text { Study start } \\
\text { date }\end{array}$ & $\begin{array}{l}\text { Age } \\
\text { range }\end{array}$ & Races/ethnicities & $\begin{array}{l}\text { Fasting } \\
\text { plasma } \\
\text { glucose }\end{array}$ & OGTT & $\begin{array}{l}\text { Fasting } \\
\text { insulin }\end{array}$ & HbAlc \\
\hline $\begin{array}{l}\text { National Health and Nutrition } \\
\text { Examination Survey } \\
{\text { (NHANES) })^{\mathrm{a}, \mathrm{e}}}\end{array}$ & $\begin{array}{l}\text { Serial cross } \\
\text { sectional }\end{array}$ & 1971 & All & All & & & & \\
\hline $\begin{array}{l}\text { National Health Interview } \\
\text { Survey (NHIS) }{ }^{\mathrm{a}, \mathrm{c}}\end{array}$ & $\begin{array}{c}\text { Serial cross } \\
\text { sectional }\end{array}$ & 1957 & All & All & SR & & & \\
\hline $\begin{array}{l}\text { National Longitudinal Survey of } \\
\text { Youth (NLSY) }\end{array}$ & Longitudinal & 1979 & $14-22$ & All & & & & \\
\hline Rancho Bernardo Study & Longitudinal & 1972 & $20+$ & Caucasian & & & & \\
\hline San Antonio Family Heart Study & Longitudinal & 1979 & $25-64$ & $\begin{array}{l}\text { Mexican American } \\
\text { and non-Hispanic } \\
\text { White }\end{array}$ & & & & \\
\hline San Luis Valley Diabetes Study & Longitudinal & 1984 & $20-74$ & $\begin{array}{l}\text { Hispanics and } \\
\text { non-Hispanic } \\
\text { Whites }\end{array}$ & & & & \\
\hline U.S. Mexico Borders Study & Cross sectional & 2001 & $18+$ & Mexicans & & & & \\
\hline $\begin{array}{l}\text { University of California San } \\
\text { Diego Filipino Health Study }\end{array}$ & Longitudinal & 1995 & $40-86$ & Filipino & & & & \\
\hline
\end{tabular}

Dark fill: data collected, white Fill: data not collected, SR: self report.

a Study is nationally representative.

${ }^{b}$ The first wave of data collection included only self-reported height and weight, but interviewer measures were added thereafter.

'Sample sizes reflect subsamples of participants who answered questions on health-related variables.

${ }^{\mathrm{d}}$ Information presented pertains to the adult sample in the 2003 NIS study.

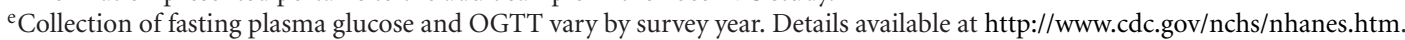

${ }^{\mathrm{f}}$ Restricted access to specific variables.

'A second phase of the study is planned, and eventually data will be publicly available.

${ }^{\text {h}}$ Self-reported heart disease, hypertension, high cholesterol are available in the following studies: CHIS, NIS, NHANES, NHIS.

iSelf-reported diabetes available in the following studies: CHIS, NIS, NHANES, NHIS.

multiple disciplines, which offer the advantage of broad, multi-disciplinary data. Many of these resources have not been extensively utilized. Given the growing awareness of the importance of chronic disease, this study demonstrates that several data sources can be used to better understand determinants of cardiometabolic diseases. Focusing on this topic in migrant populations will make it possible both to address the special needs of this population and to develop an understanding of the relative importance of place of residence, duration of exposure to environmental factors, and predispositions to disease incidence.

\section{Methods}

With both CVD and T2D at the forefront of concern in the health sciences, it is not surprising that a large number of datasets collect information on cardiometabolic health, including datasets with questions on international migration. Table 1 presents a list of the 23 studies that include variables that can be used to study CVD and T2D in immigrants in the US These are all US-based datasets that collect information on at least one measure of cardiometabolic disease and at least one indicator of international migration. The list was initially created through consultation with experts in relevant fields. We then performed extensive internet-based searches for additional datasets using the column terms listed in Table 1 . The resulting list was checked by the authors and only datasets matching the inclusion criteria were retained. The information was then verified using a combination of reviews of publications and study websites and direct contact with the principle investigator of each study.

\section{Results}

3.1. Data Sources on Migration. The most commonly used variables on migration are country of birth, year of immigration, duration of residence in the US, and parent's place of birth. Classifying individuals into foreign or native born provides insights into disease risk beyond the commonly used approach of stratification by race/ethnicity. This is important since race/ethnicity classifications combine foreign-born individuals into categories that may not accurately reflect their heritage or genetic endowments. Country of birth has not been utilized extensively in the literature, and may be a more meaningful way to group migrants, especially in the context of informing clinicians in the host community about specific cardiometabolic disease risks and prevention among immigrants. 
Additional variables, such as migration histories, citizenship status, and language use/fluency are relevant to migrant health, but are less often collected in existing data sources, and thus not highlighted in Table 1. One of the richer US data sources for the study of migration, the New Immigrant Survey, includes several such pieces of additional information on immigrants starting at their acquisition of a Green Card [8].

3.2. Data Sources on Cardiometabolic Health. There are several risk factors for developing CVD and T2D and several tests are available for diagnosing these. For research purposes, information on these is collected via clinical examination or self-report. In Table 1, we indicate which data collection mode was used in each dataset.

The most commonly collected cardiometabolic indicators are height and weight. These have the advantage of being noninvasive and can be self-reported. They also allow for the calculation of body mass index (BMI) and subsequent classification of obesity, an important risk factor for CVD and T2D [9]. Self-reported height and weight are subject to bias and several studies confirm that individuals tend to overreport height or underreport weight [10]. However, a previous study showed that foreign-born individuals selfreport height and weight at least as accurately as native-born Americans [11].

While BMI is a basic and commonly used measure, body fat distribution provides additional insights into disease risk. Therefore, measuring waist circumference (WC) is used to determine excess abdominal obesity, which is associated with an increased risk of diabetes. WC is collected less commonly, since it requires in-person measurements by trained assessors. Still, WC is an especially important measure for Asianorigin individuals, who have higher levels of abdominal adiposity even at normal BMIs [12]. More precise tests of body fat and body fat distribution tests require specialized equipment and thus are expensive and difficult to include in larger-scale studies. Table 1 shows that 12 studies measured WC and five measured total body fat percentage.

Two major risk factors associated with CVD are high blood pressure and high cholesterol, including elevated lowdensity lipoprotein (LDL). Sixteen studies measured blood pressure, a noninvasive measurement. Lipids, including total cholesterol, LDL, high-density lipoprotein (HDL), and triglycerides, can only be measured via blood samples, making them complicated and expensive measures for use in large-scale surveys. Thirteen studies measured total cholesterol.

Assessment of T2D risk factors is important for prevention of T2D, CVD, and associated comorbidities. Adults with diabetes have a two- to fourfold higher risk of CVD compared with those without diabetes [13, 14]. Diabetes risk can be clinically screened for easily using a fasting plasma glucose test assessed with a finger prick. A more sensitive test for diagnosing diabetes is the oral glucose tolerance test. However, this test is more expensive, time consuming, and invasive [15]. Fasting insulin can provide a measure of insulin resistance, a cardiometabolic condition associated with increased risk of developing diabetes [16]. Glycosylated hemoglobin (HBA1c) measures average blood glucose levels over the past 2-3 months and is most helpful when assessing diabetes management [17]. Fifteen of the studies collected one or more of these measures.

Because clinical exams can be expensive, a common alternative is to ask respondents if they have ever been diagnosed with diabetes or CVD. Although there may be biases in reporting, self-reports of hypertension, high cholesterol [18], and diabetes [19, 20] are fairly accurate in population-based studies. As shown in Table 1, 5 studies collect self-reported information on these conditions.

Age is an important correlate of cardiometabolic diseases. The majority of the studies in Table 1 focus on adults. However, nine of the studies include subjects under 18 years of age and three of the studies specifically focus on children and adolescents. Over $20 \%$ of all children in the US are immigrants or children of immigrants [21, 22 ], and may be exposed to stresses related to migration, including acculturative stress, intergenerational conflict, and discrimination and racism [23], stresses that may increase risks of developing CVD and diabetes later in life [8].

\section{Conclusions}

The aim of this narrative review was to provide a list of the main available datasets that can be used by researchers interested in the study of migration and cardiometabolic risk. The studies have some limitations. Seven of the studies are cross-sectional, offering a snapshot of health at one point in time and thus not allowing causal analysis. Results from cross-sectional data suggest that immigrants tend to weigh less upon arriving to the US compared with native-born Americans [6], but increases in both weight and obesity have been observed as duration of residence increases, specifically after living in the US for at least 10 years [2426]. Additionally, studies have found that the relationship between duration of residence and overweight/obesity varied by age at arrival, such that younger immigrants were more likely to experience overweight and obesity after living in the US compared to migrants who arrived at older ages [27]. Longitudinal data following migrants from as soon as possible after arrival for at least 5 to 10 years, with repeated data collection, preferably annually, would provide information on when behavioral and biological changes take place. In addition to simple biological measures, like BMI, information on lifestyle, including indicators of acculturation, would provide information on behavioral changes and how these changes may relate to changes in disease risks. Several longitudinal studies already exist, as shown in Table 1, but these studies do not provide sufficient information on the migration and acculturation process or sufficient information on health status during the migration and acculturation process. Longitudinal studies that identify migrants prior to migration would allow baseline assessment of demographics, weight, and lifestyle behaviors (i.e., diet and physical activity). If changes in behavior can be anticipated, interventions can be designed and implemented before detrimental behavior changes are adopted. Another limitation is that thirteen studies focus on nonrepresentative 
populations, meaning that it is not known to what extent they are generalizable.

Despite these limitations, 23 datasets already exist that can be used to explore CVD and T2D in immigrant population of the US To date, few studies explored CVD and T2D in immigrant populations [6]. This is a topic that will be of great interest, as the prevalence of cardiometabolic diseases in immigrant populations and differences in risks between people of different backgrounds are important from both epidemiologic and policy perspectives. There is preliminary evidence that immigrants have different risks of developing CVD and T2D compared with native-born individuals. These differences, and the reasons for these differences, are not well understood. While there are extensive studies on the heterogeneity in these diseases by race/ethnicity in the US, these categories do not capture the dynamics of migration and its additional influence on disease risk [7].

Collectively, the available datasets provide information on migration and cardiometabolic risk factors for most age groups and most racial-ethnic groups. Combining data from the datasets we identified here, or even from comparable studies in other countries, may offer additional opportunities to study genetic and environmental factors and interactions in health [28]. Furthermore, existing datasets serve as a starting point in developing more sophisticated or appropriate measures of migration and disease and in designing new studies in the US and other countries that also have large immigrant populations.

\section{Acknowledgments}

R. Oza-Frank was a graduate student at Emory University at the time of this study and was partly supported by a grant from the Emory University Global Health Institute. S. A. Cunningham was partly supported by an educational grant from Eli Lilly and Company and by a grant from the Emory University Global Health Institute. The authors would also like to thank the Emory University Global Health Institute for funding the NOMAD workshop. They thank Brionna Hair, B. A. and Jeremy Kane, B. S., who at the time this paper was written were graduate students at Emory University, for preliminary research support. They also thank Rob Stephenson, Ph.D., assistant professor, Emory University for comments on earlier drafts of the paper.

\section{References}

[1] World Health Organization, "Cardiovascular Diseases," 2011, http://www.who.int/cardiovascular_diseases/en/.

[2] International Diabetes Federation, "Did you know? Facts and Figures," 2011, http://www.idf.org/home/index.cfm?unode= 3B96906B-C026-2FD3-87B73F80BC22682A.

[3] P. Martin and G. Zürcher, "Managing migration: the global challenge," Population Bulletin, vol. 63, no. 1, pp. 1-20, 2008.

[4] Department of Economic and Social Affairs, Population Division. United Nations, "Trends in total migrant stock: the 2005 revision,” 2011, http://www.un.org/esa/population/publications/migration/UN_Migrant_Stock_Documentation_ 2005.pdf.
[5] A. Terrazas, J. Batalova, and V. Fan, "Frequently requested statistics on immigrants in the United States," Migration Information Source, 2011, http://www.migrationinformation .org/USfocus/display.cfm?id=649.

[6] S. A. Cunningham, J. D. Ruben, and K. M. Venkat Narayan, "Health of foreign-born people in the United States: a review," Health and Place, vol. 14, no. 4, pp. 623-635, 2008.

[7] R. Oza-Frank and K. M. Venkat Narayan, "Overweight and diabetes prevalence among US immigrants," American Journal of Public Health, vol. 100, no. 4, pp. 661-668, 2010.

[8] G. Jasso, D. Massey, M. Rosenzweig, and J. Smith, "Immigration, health, and New York City: early results based on the U.S. New Immigrant Cohort of 2003," Economic Policy ReviewFederal Reserve Bank of New York, vol. 11, pp. 127-151, 2005.

[9] P. G. Kopelman, "Obesity as a medical problem," Nature, vol. 404, no. 6778, pp. 635-643, 2000.

[10] H. Nawaz, W. Chan, M. Abdulrahman, D. Larson, and D. L. Katz, "Self-reported weight and height. Implications for obesity research," American Journal of Preventive Medicine, vol. 20, no. 4, pp. 294-298, 2001.

[11] H. Antecol and K. Bedard, "Unhealthy assimilation: why do immigrants converge to American health status levels?" Demography, vol. 43, no. 2, pp. 337-360, 2006.

[12] World Health Organization, "Appropriate body-mass index for Asian populations and its implications for policy and intervention strategies," Lancet, vol. 363, pp. 157-163, 2004.

[13] C. S. Fox, S. Coady, P. D. Sorlie et al., "Trends in cardiovascular complications of diabetes," Journal of the American Medical Association, vol. 292, no. 20, pp. 2495-2499, 2004.

[14] F. B. Hu, M. J. Stampfer, C. G. Solomon et al., "The impact of diabetes mellitus on mortality from all causes and coronary heart disease in women: 20 Years of follow-up," Archives of Internal Medicine, vol. 161, no. 14, pp. 1717-1723, 2001.

[15] National Diabetes Information Clearinghouse, "Diagnosis of Diabetes," 2011, http://diabetes.niddk.nih.gov/dm/pubs/diagnosis/.

[16] J. C. Chambers, P. Elliott, D. Zabaneh et al., "Common genetic variation near MC4R is associated with waist circumference and insulin resistance," Nature Genetics, vol. 40, no. 6, pp. 716$718,2008$.

[17] V. Mohan, R. Deepa, R. Pradeepa et al., "Association of low adiponectin levels with the metabolic syndrome-the Chennai Urban Rural Epidemiology Study (CURES-4)," Metabolism, vol. 54, no. 4, pp. 476-481, 2003.

[18] A. E. Field, E. H. Coakley, A. Must et al., "Impact of overweight on the risk of developing common chronic diseases during a 10-year period," Archives of Internal Medicine, vol. 161, no. 13, pp. 1581-1586, 2001.

[19] P. J. O'Connor, W. A. Rush, N. P. Pronk, and L. M. Cherney, "Identifying diabetes mellitus or heart disease among health maintenance organization members: sensitivity, specificity, predictive value, and cost of survey and database methods," American Journal of Managed Care, vol. 4, no. 3, pp. 335-342, 1998.

[20] S. H. Saydah, L. S. Geiss, E. Tierney, S. M. Benjamin, M. Engelgau, and F. Brancati, "Review of the performance of methods to identify diabetes cases among vital statistics, administrative, and survey data," Annals of Epidemiology, vol. 14, no. 7, pp. 507-516, 2004.

[21] M. Fix and P. JS, Immigration: Trends and Implications for Schools, The Urban Institute, Washington, DC, USA, 2003.

[22] D. J. Hernandez, "Demographic change and the life circumstances of immigrant families," Future of Children, vol. 14, no. 2, pp. 17-47, 2004. 
[23] M. Zhou, "Growing up American: the challenge confronting immigrant children and children of immigrants," Annual Review of Sociology, vol. 23, pp. 63-95, 1997.

[24] M. S. Goel, E. P. McCarthy, R. S. Phillips, and C. C. Wee, "Obesity among US immigrant subgroups by duration of residence," Journal of the American Medical Association, vol. 292, no. 23, pp. 2860-2867, 2004.

[25] D. L. Koya and L. E. Egede, "Association between length of residence and cardiovascular disease risk factors among an ethnically diverse group of United States immigrants," Journal of General Internal Medicine, vol. 22, no. 6, pp. 841-846, 2007.

[26] R. Oza-Frank and S. A. Cunningham, "The weight of US residence among immigrants: a systematic review," Obesity Reviews, vol. 11, no. 4, pp. 271-280, 2010.

[27] R. Roshania, K. M. V. Narayan, and R. Oza-Frank, "Age at arrival and risk of obesity among US immigrants," Obesity, vol. 16, no. 12, pp. 2669-2675, 2008.

[28] W. C. Willett, W. J. Blot, G. A. Colditz, A. R. Folsom, B. E. Henderson, and M. J. Stampfer, "Merging and emerging cohorts: not worth the wait," Nature, vol. 445, no. 7125, pp. 257-258, 2007. 


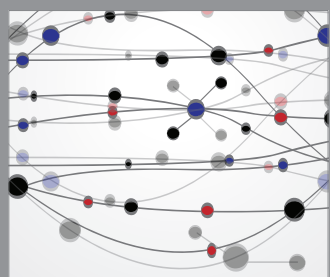

The Scientific World Journal
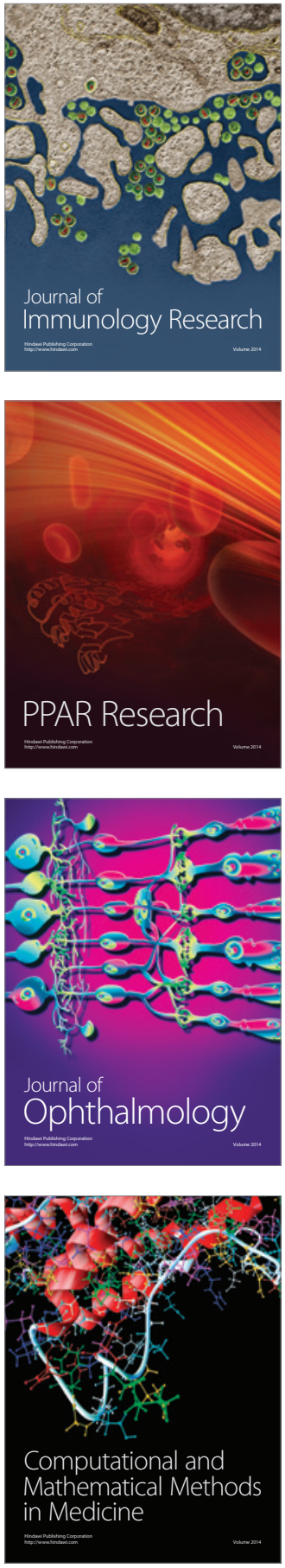

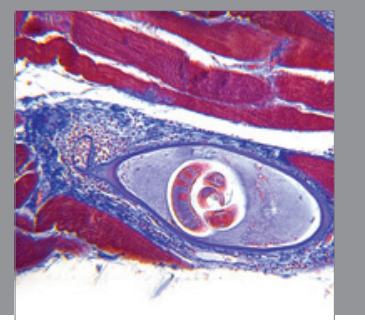

Gastroenterology

Research and Practice
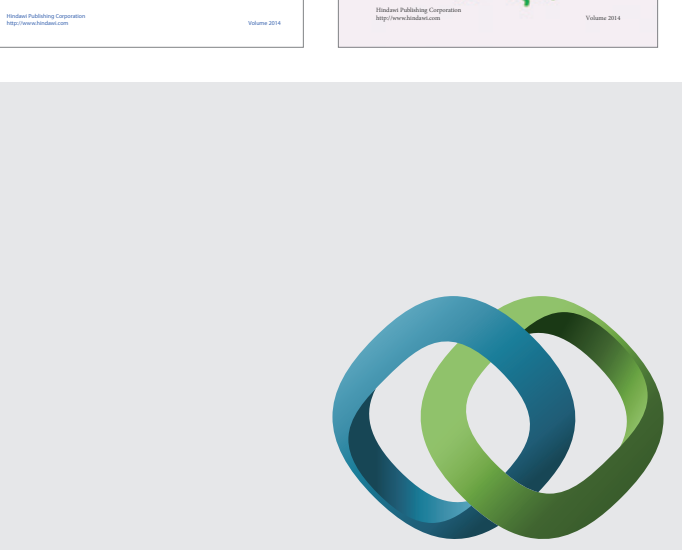

\section{Hindawi}

Submit your manuscripts at

http://www.hindawi.com
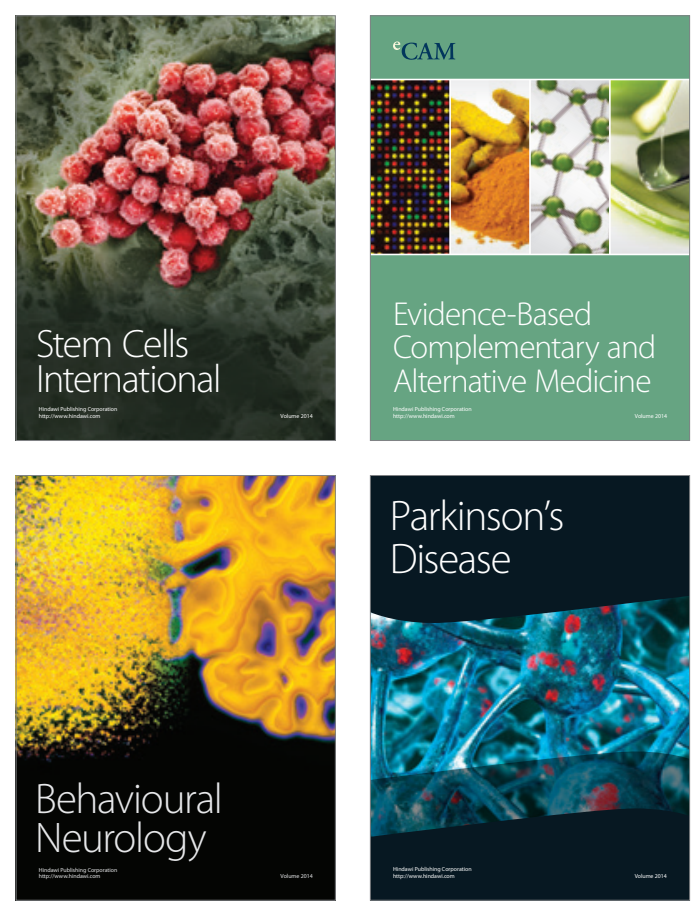

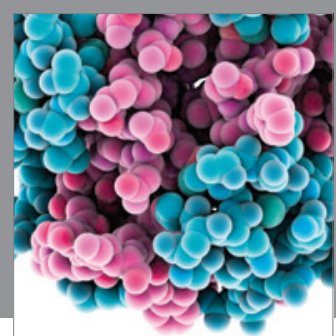

Journal of
Diabetes Research

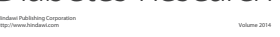

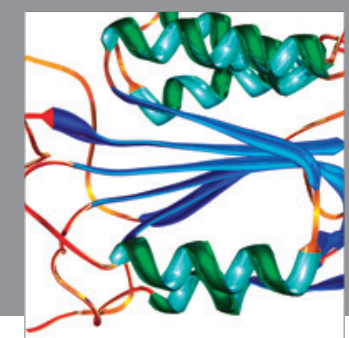

Disease Markers
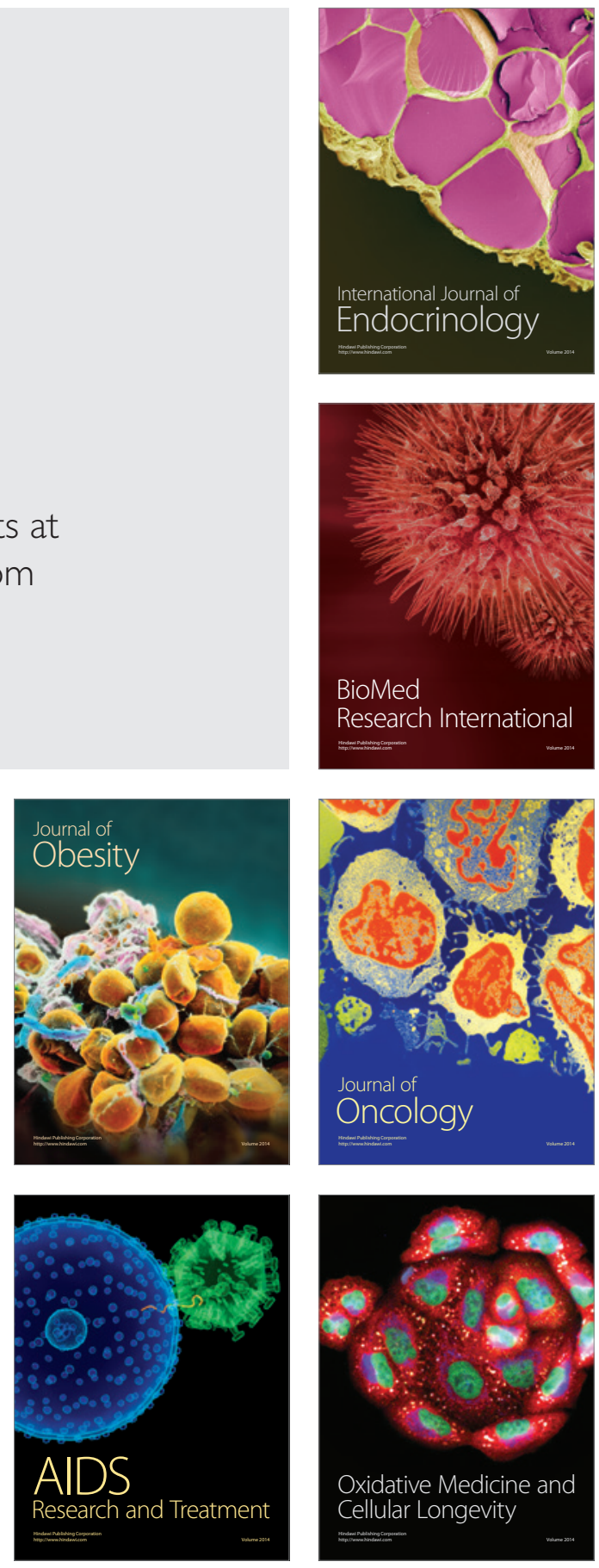